\title{
Chaotic Synchronization and Secure Communication Using Contraction Theory
}

\author{
Bharat Bhushan Sharma* and Indra Narayan Kar \\ Department of Electrical Engineering, \\ Indian Institute of Technology, Delhi, \\ Hauz Khas, New Delhi- 110016, India \\ bbs.iit@gmail.com, \\ ink@ee.iitd.ac.in
}

\begin{abstract}
Here, observer based synchronization and secure communication scheme is presented for chaotic systems. In proposed scheme, extended Kalman filter based receiver is selected for given transmitter system. The stability results are derived using virtual system concept. Observer gains for synchronization are obtained as a solution of matrix Riccati equation. For secure communication, $n$-shift ciphering algorithm is used with one of the chaotic state chosen as key. Numerical simulations are presented in the end to verify the efficacy of proposed scheme.
\end{abstract}

Keywords: Synchronization, Observer, Secure Communication.

Synchronization of chaotic systems and its application to secure communication gained momentum after the work of Pecora et al. 11. Synchronization uses output of drive system to control the slave system s.t. its output matches with that of drive system. Various synchronization schemes have been successfully applied to synchronization of chaotic systems [234]. Some observer based schemes for synchronization and secure communication can be found in 56789 . Extended Kalman filter (EKF) based approach for secure communication using multi-shift ciphering approach is given in [6]. Most of these techniques are based on Lyapunov based stability analysis. Here, we analyze synchronization of chaotic systems using EKF based observer approach. Exponential convergence of synchronizing scheme is presented using contraction framework 1011. This approach is different from Lyapunov analysis as it does not require explicit knowledge of specific attractor. In the scheme presented here, a virtual system is defined for actual \& observer system and is shown to be contracting by selecting observer gains suitably. As per contraction theory, if virtual system is contracting then its particular solutions converge to each other, exponentially. For secure communication, an encrypted information signal is transmitted to the receiver where original information is recovered using decryption algorithm. For masking the information, chaotic system states are used as carrier as well as key for improving

* Corresponding author: Bharat Bhushan Sharma, Research Scholar, Deptt. of Electrical Engineering, I.I.T. Delhi,India-110016; Tel. No. +91-11-26596133.

S. Chaudhury et al. (Eds.): PReMI 2009, LNCS 5909, pp. 549 554, 2009.

(C) Springer-Verlag Berlin Heidelberg 2009 
the security. The encryptor uses $n$-shift cipher involving a nonlinear function. At receiver, both key and carrier are reconstructed to recover original information. Speech signal transmission and retrieval is shown to justify the effectiveness of proposed strategy.

\section{Contraction Theory Results}

A nonlinear system is contracting if trajectories of perturbed system return to the nominal behaviour with an exponential convergence rate. Consider a nonlinear system

$$
\dot{\mathbf{x}}=\mathbf{f}(\mathbf{x}, t)
$$

where $\mathbf{x} \in \Re^{m}$ and $\mathbf{f}(\mathbf{x}, t)$ is continuously differentiable vector function defined as $\mathbf{f}:\left(\Re^{m} \times \Re\right) \rightarrow \Re^{m}$. Considering $\delta \mathbf{x}$ to be virtual displacement in $\mathbf{x}$, first variation of system (1) will be $\delta \dot{\mathbf{x}}=\frac{\partial \mathbf{f}(\mathbf{x}, t)}{\partial \mathbf{x}} \delta \mathbf{x}$. Defining squared distance between neighbouring trajectories as $\left(\delta \mathbf{x}^{T} \delta \mathbf{x}\right)$, one can write

$$
\frac{d}{d t}\left(\delta \mathbf{x}^{T} \delta \mathbf{x}\right)=2 \delta \mathbf{x}^{T} \frac{\partial \mathbf{f}}{\partial \mathbf{x}} \delta \mathbf{x}=2 \delta \mathbf{x}^{T} \mathbf{J} \delta \mathbf{x} \leq 2 \lambda_{m}(\mathbf{x}, t) \delta \mathbf{x}^{T} \delta \mathbf{x}
$$

Here, $\lambda_{m}(\mathbf{x}, t)$ represents the largest eigenvalue of the symmetric part of Jacobian matrix $\mathbf{J}$. If $\lambda_{m}(\mathbf{x}, t)$ is strictly uniformly negative definite (UND), then any infinitesimal length $\|\delta \mathbf{x}\|$ converges exponentially to zero. It is assured from (2) that all the solution trajectories of the system (1) converge exponentially to single trajectory, independently of the initial conditions.

Definition 1. For system in (1), a region of state space is called contracting region if Jacobian $\mathbf{J}$ is $U N D$ in that region i.e. there exists a scalar $\alpha>0, \forall \mathbf{x}, \forall t \geq$ 0 s.t. $\frac{\partial \mathbf{f}}{\partial \mathbf{x}} \leq-\alpha \mathbf{I}<\mathbf{0}$. It further implies that, $\frac{1}{2}\left(\frac{\partial \mathbf{f}}{\partial \mathbf{x}}+\frac{\partial \mathbf{f}^{T}}{\partial \mathbf{x}}\right) \leq-\alpha \mathbf{I}<\mathbf{0}$.

Lemma 1. For system (1), any trajectory which starts in a ball of constant radius centered about a given trajectory and contained at all times in a contraction region, remains in that ball and converges exponentially to given trajectory. Further, global exponential convergence to given trajectory is guaranteed if whole state space region is contracting.

Various other results related to contraction theory can be found in [101112].

\subsection{Stability Using Virtual System Theory}

Let the dynamics of a given system is rearranged as $\dot{\mathbf{x}}=\rho(\mathbf{x}, \mathbf{p}, t) \mathbf{x}$, where $\mathbf{x} \in \Re^{m}, \mathbf{p}$ is parameter vector and function $\rho(\mathbf{x}, \mathbf{p}, t) \leq \alpha \mathbf{I}<\mathbf{0}$. To show this system to be uniformly globally exponentially stable, virtual system is defined as $\dot{\mathbf{y}}=\rho(\mathbf{x}, \mathbf{p}, t) \mathbf{y}$. This system is having origin and actual system as its particular solutions. In differential framework, $\delta \dot{\mathbf{y}}=\rho(\mathbf{x}, \mathbf{p}, t) \delta \mathbf{y}$. Using UND nature of $\rho(\mathbf{x}, \mathbf{p}, t)$, states of virtual systems are ensured as contracting. It implies exponential convergence of states of actual system to each other, being a particular solution of virtual system. 
In case of observer design, virtual system is defined exactly like observer. For actual system $\dot{\mathbf{x}}_{s}=\mathbf{x}_{s}+u$, let observer be selected as $\dot{\hat{\mathbf{x}}}=\hat{\mathbf{x}}+u+L\left(\mathbf{x}_{s}-\hat{\mathbf{x}}\right)$. Here, $u$ is control input and gain $L$ is chosen s.t. $L>1$. Let the virtual system is defined as $\dot{\mathbf{x}}=\mathbf{x}+u+L\left(\mathbf{x}_{s}-\mathbf{x}\right)$. For contraction, Jacobian of virtual system should be UND. It will lead to exponential convergence of observer states $\hat{\mathbf{x}}$ to actual system states $\mathbf{x}_{s}$ as both are particular solutions of the virtual system. These results can be extended to nonlinear observer design accordingly [1].

\section{Problem Formulation}

Consider the dynamics of transmitter system be

$$
\dot{\mathbf{x}}=\mathbf{f}(\mathbf{x}, \theta, t)
$$

with output as $\mathbf{y}=\mathbf{s}(\mathbf{x}, t)$, where $\mathbf{x} \in \Re^{n}, \mathbf{y} \in \Re^{p}, \theta$ is parameter vector and $\mathbf{s}$ is linear function of measurable states. Let EKF based receiver is given as

$$
\dot{\varsigma}=\mathbf{f}(\varsigma, \theta, t)+\mathbf{K}(\varsigma, t)(\mathbf{y}-\mathbf{s}(\varsigma, t))
$$

Here, $\mathbf{K}(\varsigma, t)$ is time varying observer gain matrix and $\varsigma$ represents the estimate of actual system states $\mathbf{x}$. The output of this system is $\widehat{\mathbf{y}}=\mathbf{s}(\varsigma, t)$.

\subsection{Synchronization and Secure Communication Scheme}

In transmission-retrieval scheme shown in fig. $1, \widehat{\mathbf{x}}$ is estimate of system states $\mathbf{x}$. Transmitter state $x_{c}$ is used as carrier for modulating the information $m(t)$. Masked signal given by $S_{m}(t)=x_{c}+m(t)$ is encrypted using $n$-shift cipher algorithm [4, where key signal $k(t)$ is used $n$-times to encrypt the modulated signal. To increase the security, one of chaotic state $x_{k}$ is used as key signal. Encrypted signal is given as $e\left(S_{m}(t)\right)=\varphi\left(\ldots, \varphi\left(\varphi\left(S_{m}(t), k(t)\right), k(t)\right), \ldots, k(t)\right)$. Here, signal $S_{m}(t)$ is shifted $n$ times using nonlinear function $\varphi\left(S_{m}(t), k(t)\right)$ given as

$$
\begin{aligned}
\varphi(x, k) & =(x+k)+2 l, \quad-2 l \leq(x+k) \leq-l \\
& =(x+k), \quad-l<(x+k)<l \\
& =(x+k)-2 l, \quad l \leq(x+k) \leq 2 l
\end{aligned}
$$

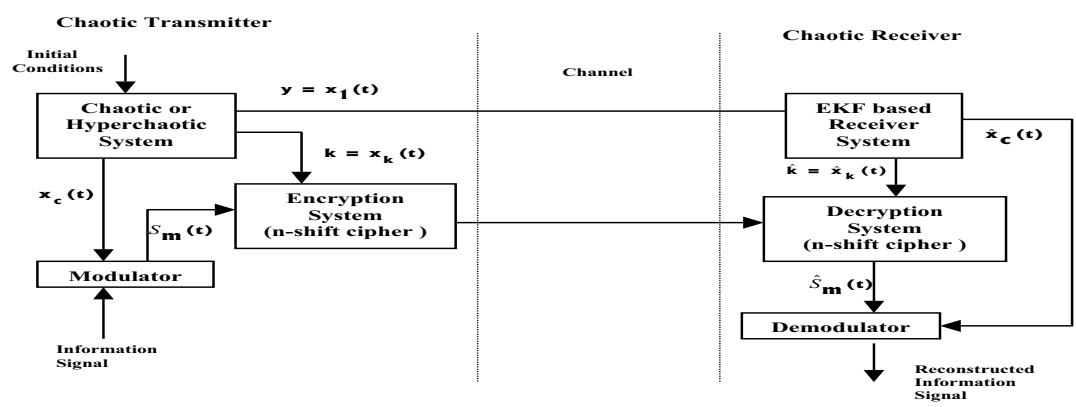

Fig. 1. Synchronization and secure communication scheme 
Parameter $l$ is chosen s.t. both $x \& k(t)$ lie in the range $(-l, l)$. Decryption rule $\widehat{S}_{m}(t)=\varphi\left(\ldots, \varphi\left(\varphi\left(S_{m}(t),-\widehat{k}(t)\right),-\widehat{k}(t)\right), \ldots,-\widehat{k}(t)\right)$, is used to recover the information. i.e. information is recovered from $\widehat{S}_{m}(t)$ as $\widehat{m}(t)=\widehat{S}_{m}(t)-\widehat{x}_{c}$.

\subsection{Stability Analysis for Observer Based Communication Scheme}

For the systems in (3) \& (4), let observer gain $\mathbf{K}(\varsigma, t)$ is defined as

$$
\mathbf{K}(\varsigma, t)=\mathbf{P}(t) \mathbf{C}^{T}(\varsigma, t) \mathbf{S}^{-1}
$$

For transmitter (3), define matrices $\mathbf{A}(\varsigma, t)=\left.\frac{\partial \mathbf{f}(\mathbf{x}, \theta, t)}{\partial \mathbf{x}}\right|_{\mathbf{x}=\varsigma}$ and $\mathbf{C}(\varsigma, t)=\left.\frac{\partial \mathbf{s}(\mathbf{x}, t)}{\partial \mathbf{x}}\right|_{\mathbf{x}=\varsigma}$. To obtain $\mathbf{K}(\varsigma, t)$, time varying uniformly positive definite matrix $P(t)$ is determined as a solution of matrix differential Riccati equation:

$$
\dot{\mathbf{P}}(t)=\mathbf{A} \mathbf{P}(t)+\mathbf{P}(t) \mathbf{A}^{T}-\mathbf{P}(t) \mathbf{C}^{T} \mathbf{S}^{-1} \mathbf{C P}(t)+\mathbf{Q}
$$

Here, $\mathbf{A}=\mathbf{A}(\varsigma, t), \mathbf{C}=\mathbf{C}(\varsigma, t)$ and covariance matrices $\mathbf{Q} \& \mathbf{S}^{-1}$ are assumed to be constant symmetric positive definite (s.p.d.) matrices.

Assumption 1: In the Riccati equation (7), the solution matrix $\mathbf{P}(t)$ is assumed to be uniformly positive definite and bounds on $\mathbf{P}(t)$ are defined as $P_{\min } \leq$ $\mathbf{P}(t) \leq P_{\max }$ where $P_{\min }$ and $P_{\max }$ are positive real numbers.

Synchronization results are presented in the form of a lemma here [12.

Lemma 2. The EKF based receiver system (3) with gains in (6) gets synchronized with transmitter system (3), exponentially under the assumption 1.

Proof: Let the virtual system for the systems in (3) \& (4) is defined as

$$
\dot{\mathbf{x}}_{v}=\mathbf{f}\left(\mathbf{x}_{v}, \theta, t\right)+\mathbf{K}(\varsigma, t)\left(\mathbf{y}-\mathbf{s}\left(\mathbf{x}_{v}, t\right)\right)
$$

with output as $\mathbf{y}_{v}=\mathbf{s}\left(\mathbf{x}_{v}, t\right)$. In differential framework, one can write,

$$
\left.\delta \dot{\mathbf{x}}_{v}=\left[\frac{\partial}{\partial \mathbf{x}_{v}} \mathbf{f}\left(\mathbf{x}_{v}, \theta, t\right)-\mathbf{K}(\varsigma, t) \frac{\partial}{\partial \mathbf{x}_{v}} \mathbf{s} \mathbf{x}_{v}, t\right)\right] \delta \mathbf{x}_{v}=[\mathbf{A}-\mathbf{K}(\varsigma, t) \mathbf{C}] \delta \mathbf{x}_{v}
$$

Time derivative of weighted square distance $\left(\delta \mathbf{x}_{v}^{T} \mathbf{M} \delta \mathbf{x}_{v}\right)$ will be

$$
\frac{d}{d t}\left(\delta \mathbf{x}_{v}^{T} \mathbf{M} \delta \mathbf{x}_{v}\right)=\delta \mathbf{x}_{v}^{T} \mathbf{M} \delta \dot{\mathbf{x}}_{v}+\delta \mathbf{x}_{v}^{T} \frac{d}{d t} \mathbf{M} \delta \mathbf{x}_{v}+\delta \dot{\mathbf{x}}_{v}^{T} \mathbf{M} \delta \mathbf{x}_{v}
$$

By defining $\mathbf{M}=P^{-1}$ and simplifying using (9), one can get

$$
\frac{d}{d t}\left(\delta \mathbf{x}_{v}^{T} \mathbf{P}^{-1} \delta \mathbf{x}_{v}\right)=\delta \mathbf{x}_{v}^{T} \mathbf{P}^{-1}\left[\mathbf{A}_{c} \mathbf{P}-\dot{\mathbf{P}}+\mathbf{P A}_{c}^{T}\right] \mathbf{P}^{-1} \delta \mathbf{x}_{v}
$$

where $\mathbf{A}_{c}=(\mathbf{A}-\mathbf{K C})$. Using equation (7) and observer gain $\mathbf{K}(\varsigma, t)$, we get

$$
\begin{aligned}
\frac{d}{d t}\left(\delta \mathbf{x}_{v}^{T} \mathbf{P}^{-1} \delta \mathbf{x}_{v}\right) & =-\delta \mathbf{x}_{v}^{T} \mathbf{C}^{T} \mathbf{S}^{-1} \mathbf{C} \delta \mathbf{x}_{v}-\delta \mathbf{x}_{v}^{T} \mathbf{P}^{-1} \mathbf{Q} \mathbf{P}^{-1} \delta \mathbf{x}_{v} \\
\Rightarrow \frac{d}{d t}\left(\delta \mathbf{x}_{v}^{T} \mathbf{P}^{-1} \delta \mathbf{x}_{v}\right) & =-\delta y_{v}^{T} \mathbf{S}^{-1} \delta y_{v}-\delta \mathbf{x}_{v}^{T} \mathbf{P}^{-1} \mathbf{Q} \mathbf{P}^{-1} \delta \mathbf{x}_{v}
\end{aligned}
$$


This is because differential output $\delta y_{v}=\mathbf{C} \delta \mathbf{x}_{v}$. As $\mathbf{S}^{-1}$ is constant symmetric positive definite matrix, so equation (11) can be reduced to

$$
\frac{d}{d t}\left(\delta \mathbf{x}_{v}^{T} \mathbf{P}^{-1} \delta \mathbf{x}_{v}\right) \leq-\delta \mathbf{x}_{v}^{T} \mathbf{P}^{-1} \mathbf{Q} \mathbf{P}^{-1} \delta \mathbf{x}_{v} \leq-\kappa \delta \mathbf{x}_{v}^{T} \mathbf{P}^{-1} \delta \mathbf{x}_{v}
$$

where $\kappa=q_{\min } / P_{\max }, q_{\min }$ is smallest eigenvalue of matrix $\mathbf{Q}$ and $P_{\max }$ is largest eigenvalue of $\mathbf{P}$. It clearly shows contracting nature of virtual system. Hence, receiver states converge to transmitter states, exponentially.

\section{Numerical Simulations}

To analyze the synchronization in transmitter-receiver configuration, consider the chaotic Chen system to be transmitter having dynamics as

$$
\begin{aligned}
& \dot{x}_{1}=-a x_{1}+a x_{2} \\
& \dot{x}_{2}=-x_{1} x_{3}+c x_{2}+(c-a) x_{1} \\
& \dot{x}_{3}=x_{1} x_{2}-b x_{3}
\end{aligned}
$$

Lt the output is given as $y=x_{1}$. Here, $a, b \& c$ are positive real constants of the system. System in (13) exhibits chaotic behaviour for $a=40, c=31$ and $b=3$. For EKF based receiver system, output is taken as $\widehat{y}=\varsigma_{1}$ and matrix $\mathbf{A}$ and $\mathbf{C}$ are evaluated as discussed in section 2.2. Initial conditions for transmitter and receiver are taken as $\mathbf{x}(0)=\left(\begin{array}{lll}2 & 1 & 2\end{array}\right)^{T}$ and $\varsigma(0)=\left(\begin{array}{lll}1 & 2 & 1\end{array}\right)^{T}$, respectively.

(a)

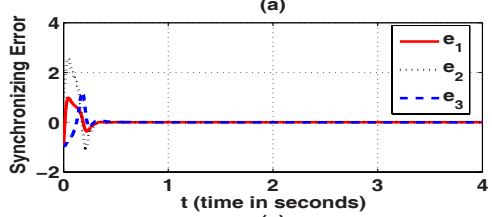

(c)

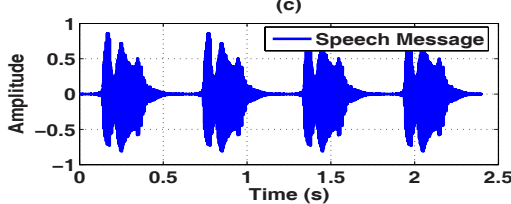

(e)

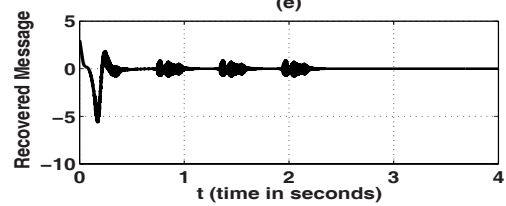

(b)

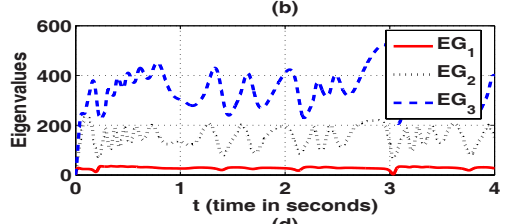

(d)

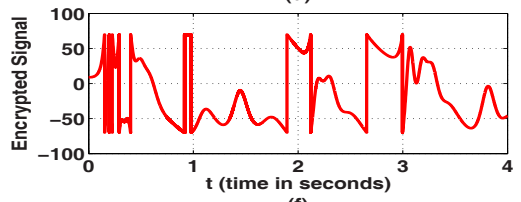

(f)

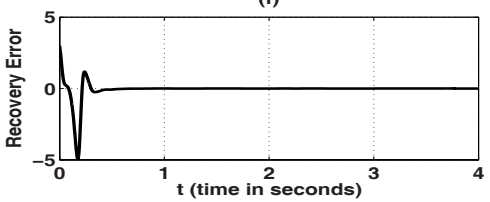

Fig. 2. Synchronization and secure communication: (a) Synchronization error; (b) Variation of eigenvalues of $P(t)$; (c) Speech message; (d) Encrypted signal; (e) Recovered message \& (f) Message recovery error 
$\mathbf{P}$ and $\mathbf{Q}$ are initialized as diagonal matrices with $P_{i, i}=1 \times 10^{-4}$ and $Q_{i, i}=$ $0.35 \times 10^{4}$, respectively. Fig. 2 gives various plots related to synchronization and communication scheme. Multiple envelopes of a speech signal corresponding to word "HELLO" is transmitted after modulating it with carrier $x_{2}$. The signal is sampled at $16 \mathrm{KHz}$ frequency and is having total duration of 0.6 seconds. State $x_{3}$ is used as the key signal for encryption. These figures clearly show the effectiveness of proposed approach in secure transmission and recovery of the message signal.

\section{Conclusion}

Synchronization and secure communication scheme for chaotic systems is presented where exponential stability is shown using virtual system concept in quite a simple manner. Systems once synchronized, can be used for information transmission and recovery. For secure communication, multi-shift key algorithm for encryption is utilized which uses one of chaotic state as key for encryption.

\section{References}

1. Pecora, L.M., Carroll, T.L.: Synchronization in Chaotic Systems. Phys. Rev. Lett. 64, 821-824 (1990)

2. Carroll, T.L., Pecora, L.M.: Synchronizing Chaotic Circuits. IEEE Trans. Circ. Syst.-I 38(4), 453-456 (1991)

3. Yang, L.X., Chu, Y.D., Zhang, J.G., Li, F.L., Chang, Y.X.: Chaos Synchronization in Autonomous Chaotic System via Hybrid Feedback Control. Chaos, Solitons and Fractals 41(1), 214-223 (2009)

4. Yang, T., Wu, C.W., Chua, L.O.: Cryptography based on Chaotic Systems. IEEE Trans. on Circ. Syst.-I 44(5), 469-472 (1997)

5. Boutayeb, M., Darouach, M., Rafaralahy, H.: Generalized State-space Observers for Chaotic Synchronization with Applications to Secure Communication. IEEE Trans. Circ. Syst.-I 49(3), 345-349 (2002)

6. Fallahi, K., Raoufi, R., Khoshbin, H.: An Application of Chen System for Secure Chaotic Communication based on Extended Kalman Filter and Multi-Shift Cipher Algorithm. Comm. in Nonlinear Science and Num. Sim. 13(4), 763-781 (2008)

7. Grassi, G., Mascolo, S.: Nonlinear Observer Design to Synchronize Hyperchaotic Systems via a Scalar Signal. IEEE Trans. Circ. Syst.-I 44(10), 1011-1014 (1997)

8. Liao, T.L., Huang, N.S.: An Observer based Approach for Chaotic Synchronization and Secure Communication. IEEE Trans. Circ. Syst.-I 46(9), 1144-1149 (1999)

9. Morgul, O., Solak, E.: Observer based Synchronization of Chaotic Systems. Phys. Rev. E 54(5), 4803-4811 (1996)

10. Lohmiller, W.: Contraction Analysis of Nonlinear Systems, Ph.D. Thesis, Department of Mechanical Engineering, MIT (1999)

11. Lohmiller, W., Slotine, J.J.E.: On Contraction Analysis for Nonlinear Systems. Automatica 34(6), 683-696 (1998)

12. Jouffroy, J., Slotine, J.J.E.: Methodological Remarks on Contraction Theory. In: IEEE Conf. on Decision Control, Atlantis, Bahamas, pp. 2537-2543 (2004) 